\title{
TWO DIMENSIONAL OPTIMAL TRANSPORTATION PROBLEM FOR A DISTANCE COST WITH A CONVEX CONSTRAINT**
}

\author{
Ping Chen ${ }^{1,2}$, Feida Jiang ${ }^{1}$ and Xiaoping Yang ${ }^{1}$
}

\begin{abstract}
We first prove existence and uniqueness of optimal transportation maps for the Monge's problem associated to a cost function with a strictly convex constraint in the Euclidean plane $\mathbb{R}^{2}$. The cost function coincides with the Euclidean distance if the displacement $y-x$ belongs to a given strictly convex set, and it is infinite otherwise. Secondly, we give a sufficient condition for existence and uniqueness of optimal transportation maps for the original Monge's problem in $\mathbb{R}^{2}$. Finally, we get existence of optimal transportation maps for a cost function with a convex constraint, i.e. $y-x$ belongs to a given convex set with at most countable flat parts.
\end{abstract}

Mathematics Subject Classification. 49Q20, 49J45.

Received May 11, 2012. Revised December 30, 2012.

Published online July 4, 2013.

\section{INTRODUCTION}

The theory of mass transportation goes back to the original works by Monge in 1781 [16] and later by Kantorovich in 1942 [13]. The Monge's optimal transportation problem can be rephrased as follows:

$$
\min _{T_{\sharp} \mu=\nu} \int_{\mathbb{R}^{n}} c(x, T(x)) \mathrm{d} \mu(x),
$$

where $\mu$ and $\nu$ are given probability measures on $\mathbb{R}^{n}$, and the admissible set is given by all measurable maps $T: \mathbb{R}^{n} \rightarrow \mathbb{R}^{n}$ pushing forward $\mu$ to $\nu$ which is usually denoted by $T_{\sharp} \mu=\nu$, i.e.

$$
\nu(A)=\mu\left(T^{-1}(A)\right) \text { for all } A \subset \mathbb{R}^{n} \text { measurable }
$$

and in such a way that $T$ minimizes (1.1). In (1.1) $c: \mathbb{R}^{n} \times \mathbb{R}^{n} \rightarrow \mathbb{R}$ is a given cost function. The integral is called total cost of transportation, and the value of the optimization is called optimal cost of transportation. In the original formulation given by Monge in 1781, the cost function was the Euclidean distance and the measures $\mu, \nu$ were supposed to be absolutely continuous with respect to $\mathcal{L}^{n}$ and supported on two disjoint compact

Keywords and phrases. Optimal transportation map, convex constraint, Monge transportation problem.

* Supported by National Natural Science Foundation of China (No. 11071119, No. 11126237, No. 11101001).

1 School of Science, Nanjing University of Science and Technology, Nanjing 210094, P.R. China.

chenping200517@126.com; jfd2001@163.com; yangxp@mail.njust.edu.cn

2 School of Mathematics and Computer Science, Anhui Normal University, Wuhu 241000, P.R. China. 
subsets in $\mathbb{R}^{n}$. When the transportation condition $T_{\sharp} \mu=\nu$ is satisfied, we say that $T$ is a transportation map, and if $T$ minimizes also the cost of transportation we call it an optimal transportation map. Recently a renewed interest for this theory arose in different areas of applied mathematics like economic sciences, fluid mechanics, shape optimization, image and data compression as well as in geometric functional analysis and large deviation theory.

The main difficulty in attacking the Monge problem is due to the facts that the objective functional $\int_{\mathbb{R}^{n}} c(x, T(x)) \mathrm{d} \mu(x)$ is nonlinear with respect to $T$ of the condition $T_{\sharp} \mu=\nu$, and the admissible set of transportation maps does not possess the right compactness properties to apply the direct method in the calculus of variations. In 1940s, Kantorovich proposed in $[13,14]$ a notion of weak solutions of the transportation problem.

Denote by $\mathcal{P}\left(\mathbb{R}^{n} \times \mathbb{R}^{n}\right)$ the set of all Borel probability measures on $\mathbb{R}^{n} \times \mathbb{R}^{n}$.

The set of transportation plans between $\mu$ and $\nu$ is defined as

$$
\Pi(\mu, \nu)=\left\{\gamma \in \mathcal{P}\left(\mathbb{R}^{n} \times \mathbb{R}^{n}\right): \pi_{\sharp}^{1} \gamma=\mu, \pi_{\sharp}^{2} \gamma=\nu\right\},
$$

where $\pi^{i}: \mathbb{R}^{n} \times \mathbb{R}^{n} \rightarrow \mathbb{R}^{n}, \pi^{i}\left(x_{1}, x_{2}\right)=x_{i}, i=1,2$. In some versions, those admissible transportation plans are also said to have marginals $\mu$ and $\nu$. The set of admissible transportation plans is always nonempty, since it contains at least the tensor product $\mu \times \nu[1]$.

Given $c: \mathbb{R}^{n} \times \mathbb{R}^{n} \rightarrow[0,+\infty]$ a lower semicontinuous cost function, the Kantorovich's optimal transportation problem is defined as:

$$
\min \left\{\int_{\mathbb{R}^{n} \times \mathbb{R}^{n}} c(x, y) \mathrm{d} \pi(\mu, \nu): \pi \in \Pi(\mu, \nu)\right\} .
$$

This minimization problem introduced by Kantorovich is the relaxed formulation of the Monge problem [19,20]. The existence of a minimizer comes from the direct methods in the calculus of variations. We call the solutions to (1.2) optimal transportation plans.

Kantorovich's approach [13,14] leads to a dual formulation of the optimal transportation problem. The dual formulation is one of qualitative descriptions of optimal transportation problems. In addition, the dual theory is a main approach to prove existence of optimal maps. We refer to $[1,2,19,20]$.

In this paper, we will focus on a Euclidean distance cost function with the following convex constraint:

$$
c(x, y)=\left\{\begin{array}{cl}
|x-y|, & \text { if } y-x \in C, \\
+\infty, & \text { otherwise }
\end{array}\right.
$$

in $\mathbb{R}^{2}$, where $C$ is a closed convex set in $\mathbb{R}^{2}$. The convex constraint here means that the point $y-x$ belongs to a given convex set $C$. And the corresponding optimal transportation problem is called a convex constrained optimal transportation problem. Recently, constrained optimal transportation problems have attracted much attention. In fact, the $L^{\infty}$ problem studied in [8] can also be considered as a constrained problem (if the minimal $L^{\infty}$ norm $\|T(x)-x\|_{L^{\infty}}$ is $M$, this means that we could consider maps satisfying $\left.T(x)-x \in \overline{B(0, M)}\right)$. Carlier-De PascaleSantambrogio studied transportation problems with convex cost functions (not necessary strictly convex) [5]. The strategy is to reduce these problems to some convex constrained cases. Jimenez-Santambrogio [12] studied a convex constrained optimal transportation problem with a quadratic cost function, which was a problem with a strictly convex cost function. It is natural to consider a constrained optimal transportation problem with the original Monge's cost function, which is a classical problem with a distance cost function lack of strict convexity. Historically, Sudakov [17] and Bianchini-Cavalletti [3] studied existence of solutions of the original Monge problem in the case $X=Y=\mathbb{R}^{2}$; higher dimensional problems were investigated by Evans-Gangbo [11], Caffarelli-Feldman-McCann [4], and Trudinger-Wang [18]. Simpler approaches were proposed by ChampionDe Pascale [7] and Bianchini-Cavalletti [3] more recently. In this paper we shall establish existence results for two-dimensional transportation problems with cost function (1.3).

To illustrate the difference between our setting and the usual distance cost function, we give an example. 
Example 1.1. Let $u_{1}<u_{2}<u_{3}<u_{4} \in \mathbb{R}$ be four points with the following distances: $\left|u_{1}-u_{2}\right|=\left|u_{2}-u_{3}\right|=$ $\left|u_{3}-u_{4}\right|=1$. Set $\mu=\delta_{u_{1}}+\delta_{u_{2}}$ and $\nu=\delta_{u_{3}}+\delta_{u_{4}}$. There are two optimal transportation maps for the Monge problem (1.1) with a Euclidean distance cost function. $T u_{1}=u_{3}, T u_{2}=u_{4}$ and $T u_{1}=u_{4}, T u_{2}=u_{3}$ are both optimal transportation maps, and the optimal cost of transportation is 4 .

But the second map is not optimal for the Monge problem (1.1) with the cost function $c$ defined by (1.3) where $C$ is the closed ball $\overline{B(0, r)},(2<r<3)$. Since the distance between $u_{1}$ and $u_{4}$ is 3 , then $u_{4}-u_{1} \notin C$. As a result, the cost is infinite if the mass located at $u_{1}$ is transported to location $u_{4}$. The unique optimal transportation map for cost function (1.3) is the above first map in the case of a distance cost function.

On the other hand, if a convex constraint $C$ is large enough, for example, if we take $C=\mathbb{R}^{2}$, the convex constrained optimal transportation problem with cost function (1.3) is the same as the original Monge problem. For the original Monge cost function, the minimizers of (1.1) are not generally unique, even on the line $X=$ $Y=\mathbb{R}$. In our paper, we prove not only existence but also uniqueness of optimal transportation maps of strictly convex constrained optimal transportation problems.

When we add a constraint, existence of solutions of the dual formulation is not guaranteed: the usual proof of existence of solutions to the dual problem requires continuity or finiteness of cost functions $[19,20]$. The dual theory seems to be invalid in our case.

Recently, Champion-De Pascale-Juutinen studied some properties of transportation plans based on the density theory of measures in [8]. Using these properties, Champion-De Pascale proved existence of solutions to the Monge problem in $\mathbb{R}^{n}[6,7]$. By extending these properties of transportation plans to the Heisenberg group, De Pascale-Rigot proved existence of optimal transportation maps for the Monge problem with a C-C distance cost function in the Heisenberg group [10]. In this paper, we prove existence and uniqueness results of the Monge problem in $\mathbb{R}^{2}$, where the cost function is defined as (1.3). Our tools include the properties of transportation plans, density theory, c-cyclical monotonicity. We study convex constrained optimal transportation problems in $\mathbb{R}^{n}$ in [9] using other different tools such as variational approximation, $L^{\infty}$-estimate of an displacement interpolation and so on. In the case of $\mathbb{R}^{n}$, we can only prove existence of optimal transportation maps. Existence of 2-dimensional case can be seen as a special result of that of n-dimensional case. Uniqueness of 2-dimensional case comes from further assumptions on initial transportation measures.

Our paper is organized as follows: in Section 2, we state some preliminaries in optimal transportation theory, convex sets and properties of transportation plans based on density theory. In Section 3, we address a convex constrained optimal transportation problem in $\mathbb{R}^{2}$ where the cost function is the Euclidean distance with a strictly convex constraint. We prove existence and uniqueness of optimal transportation maps. We also give a sufficient condition to get existence and uniqueness results for the original Monge's problem in $\mathbb{R}^{2}$. In Section 4, we consider the case of a distance cost function with a convex constraint (but not strictly convex). We show existence of optimal transportation maps.

\section{Preliminaries}

In this section, we recall some general facts about optimal transportation, convex analysis and density theory.

Let us recall a notion which characterizes optimality in the Kantorovich's problem.

Definition 2.1 ([15] c-cyclically monotone sets). $S \subset \mathbb{R}^{n} \times \mathbb{R}^{n}$ is called c-cyclically monotone if and only if all $k \in \mathbb{N}$ and $\left(x_{1}, y_{1}\right), \ldots \ldots\left(x_{k}, y_{k}\right) \in S$ satisfy

$$
\sum_{i=1}^{k} c\left(x_{i}, y_{i}\right) \leq \sum_{i=1}^{k} c\left(x_{i}, y_{\sigma(i)}\right)
$$

for each permutation $\sigma$ of $k$ letters. 
Theorem 2.2. If the cost function $c(x, y)$ is lower semicontinuous and the optimal cost $\inf _{\pi \in \Pi(\mu, \nu)} \int c(x, y) \mathrm{d} \pi$ is finite, then optimality of $\gamma \in \Pi(\mu, \nu)$ implies $\gamma$ is concentrated on a c-cyclically monotone set $\Gamma$ (see [20], Thm. 5.10).

We say that a transportation plan $\gamma \in \Pi(\mu, \nu)$ is induced by a transportation map if there exists a $\mu$ measurable map $T: \mathbb{R}^{n} \rightarrow \mathbb{R}^{n}$ such that $(i d \times T)_{\sharp} \mu=\gamma$, where $(i d \times T)(x)=(x, T x)$. Such a map is automatically a transportation map between $\mu$ and $\nu$. Another equivalent statement of $(i d \times T)_{\sharp} \mu=\gamma$ is that:

$$
\int_{\mathbb{R}^{n} \times \mathbb{R}^{n}} \phi(x, y) \mathrm{d} \gamma(x, y)=\int_{\mathbb{R}^{n}} \phi(x, T x) \mathrm{d} \mu(x) \quad \forall \phi \in \mathcal{C}_{0}\left(\mathbb{R}^{n} \times \mathbb{R}^{n}\right) .
$$

We recall that if a transportation plan is concentrated on a $\gamma$-measurable graph then $\gamma$ is induced by a transportation map. By measurable graph we mean a set of a form $\Gamma=\left\{(x, T x) \in \mathbb{R}^{n} \times \mathbb{R}^{n}: x \in S\right\}$ for some measurable sets $S$ and some measurable maps $T$.

Theorem 2.3 ([10] Optimal transportation plans versus optimal transportation maps).

1. Assume that $\gamma$ is an optimal transportation plan of the Kantorovich's problem (1.2) and that $\gamma$ is induced by a transportation map $T$. Then $T$ is an optimal transportation map of (1.1).

2. Assume that any optimal transportation plan of the Kantorovich's transportation problem (1.2) is induced by a transportation map. Then there exists a unique optimal transportation map for the transportation problem (1.1).

Next we recall some definitions about convex sets.

Definition 2.4 [12]. Consider a convex set $C \subset \mathbb{R}^{n}$.

1. If $x_{0}$ is any point of $C$, we call dimension of $C$ the dimension of $\operatorname{span}\left(C-x_{0}\right)$, i.e. the dimension of the smallest affine space containing $C$. This number is denoted by $\operatorname{dim}(C)$ and does not depend on the choice of $x_{0}$. The interior and boundary of $C$ in the canonical topology associated to the Euclidean distance in such an affine space are called relative interior and relative boundary and denoted by $r i C$ and $r \partial C$.

2. A convex subset $F \subset C$ containing more than one point is called a straight part of $C$ if it is contained in relative boundary $r \partial C$.

3. A subset $F$ of a convex set $C$ is called a maximal flat part of $C$ if it is maximal for the inclusion among straight parts of $C$.

4. A subset $F$ of a convex set $C$ is called a flat part of $C$ if there is a finite chain of convex sets $F=F_{1} \subset F_{2} \subset$ $\ldots \subset F_{k-1} \subset F_{k}=C \quad(k \geq 2)$ such that, for each $i=1, \ldots, k-1, F_{i}$ is a maximal flat part of $F_{i+1}$. Notice that in this case one has $\operatorname{dim}\left(F_{1}\right)<\operatorname{dim}\left(F_{2}\right)<\ldots<\operatorname{dim}\left(F_{k}\right)$. In particular, for every flat part $F$ of $C$ we have $1 \leq \operatorname{dim}(F) \leq(d-1)$.

5. A set $C \subset \mathbb{R}^{d}$ is said to be strictly convex if it has no flat parts.

Finally we recall some properties of transportation plans. These properties are proved based on the density theory. The definitions and techniques detailed below were first introduced in [8] and extended to more general settings, for instance in any separable doubling metric measure space [10].

Definition $2.5[6-8]$. Let $y \in \mathbb{R}^{n}, r>0$ and let $\gamma \in \Pi(\mu, \nu)$ be a transportation plan, we define

$$
\gamma^{-1}(\overline{B(y, r)}):=\pi^{1}\left(\left(\mathbb{R}^{n} \times \overline{B(y, r)}\right) \cap \text { spt } \gamma\right) \text { where } \pi^{1}: \begin{gathered}
\mathbb{R}^{n} \times \mathbb{R}^{n} \rightarrow \mathbb{R}^{n} \\
(x, y) \mapsto x .
\end{gathered}
$$

In other words, $\gamma^{-1}(\overline{B(y, r)})$ is the set of points whose mass is partially or completely transported to $\overline{B(y, r)}$. We recall that any optimal plan $\gamma$ is concentrated on a c-cyclical monotone set $\Gamma$ when the cost function is lower semicontinuous. The reason to deal with $\sigma$-compact sects $\Gamma$, in the above definition as well as in 
the following, is that the projection $\pi^{1}(\Gamma)$ is also $\sigma$-compact, and in particular is a Borel set. Then Definition 2.5 can be refined as:

Definition $2.6[6-8]$. let $\gamma \in \Pi(\mu, \nu)$ be a transportation plan and $\Gamma$ a $\sigma$-compact set on which $\gamma$ is concentrated. For $y \in \mathbb{R}^{n}$ and $r>0$, we define

$$
\gamma^{-1}(\overline{B(y, r)}):=\pi^{1}\left(\left(\mathbb{R}^{n} \times \overline{B(y, r)}\right) \cap \Gamma\right) .
$$

Also, we need the following lemmas.

Lemma 2.7 [6-8]. Assume that $\mu \ll \mathcal{L}^{n}$. Let $\gamma \in \Pi(\mu, \nu)$, and $\Gamma$ a set on which $\gamma$ is concentrated. Then there exists a $\sigma$-compact subset $R(\Gamma)$ of $\Gamma \cap$ spt $\gamma$ on which $\gamma$ is concentrated and such that for any element $\left(x_{0}, y_{0}\right)$ in $R(\Gamma)$, the point $x_{0}$ is a Lebesgue point of $\gamma^{-1}\left(\overline{B\left(y_{0}, r\right)}\right)$ for all $r>0$, i.e.:

$$
\lim _{\epsilon \rightarrow 0} \frac{\mathcal{L}^{n}\left(\gamma^{-1}\left(\overline{B\left(y_{0}, r\right)}\right) \cap B\left(x_{0}, \epsilon\right)\right)}{\mathcal{L}^{n}\left(B\left(x_{0}, \epsilon\right)\right)}=1 .
$$

Lemma 2.8 [12]. Let $\left(x_{0}, y_{0}\right)$ in $R(\Gamma)$, let $r>0, \delta \in(0,1)$ and $\xi$ a unit vector in $\mathbb{R}^{n}$, then for any $\epsilon>0$, the following set has positive Lebesgue measure:

$$
\gamma^{-1}\left(\overline{B\left(y_{0}, r\right)}\right) \cap B\left(x_{0}, \epsilon\right) \cap C\left(x_{0}, \xi, \delta\right)
$$

where the set $C\left(x_{0}, \xi, \delta\right)$ is the following convex cone:

$$
\left\{x:\left\langle x-x_{0}, \xi\right\rangle>(1-\delta)\left|x-x_{0}\right|\right\}
$$

(notice that it is an open cone, with $x_{0} \notin C\left(x_{0}, \xi, \delta\right)$ ).

Lemma 2.9 [12]. Let $C$ be a convex set in $\mathbb{R}^{n}, x \in C$ and $\xi$ a unit vector, $\eta>0$ such that $x+\eta \xi \in \operatorname{Int}(C)$. Let $0<\eta^{\prime}<\eta$ be fixed. Then there exist $r>0$ and $\delta \in(0,1)$ such that

$$
\forall y \in B(x, r) \cap C, \quad\left(C(y, \xi, \delta) \cap \overline{B\left(y, \eta^{\prime}\right)}\right) \subset \operatorname{Int}(C) .
$$

The direction $\xi$ is called an entering direction at the point $x$.

\section{Cost Functions With strictly COnvex CONStRaints}

One of the main results of this paper is to prove existence and uniqueness of minimizers of the Monge problem (1.1). We consider the optimal transportation problem with cost function (1.3), where $C$ is a strictly convex closed subset in $\mathbb{R}^{2}$.

Theorem 3.1. Consider the Monge-Kantorovich problem with the cost function given by (1.3) with a closed and strictly convex set $C$. Let $\mu$ and $\nu$ be probability measures in $\mathbb{R}^{2}$, assume that

1. $\mu \ll \mathcal{L}^{2}$,

2. for all $\gamma \in \Pi(\mu, \nu)$, and $\gamma$-a.e. $(x, y),\left(x, y^{\prime}\right), y \neq y^{\prime}$ satisfying $y-x \in C$ and $y^{\prime}-x \in C$, then $x, y, y^{\prime}$ do not lie in a single line.

3. there exists $\pi \in \Pi(\mu, \nu)$ such that $\int_{\mathbb{R}^{2} \times \mathbb{R}^{2}} c(x, y) \mathrm{d} \pi(x, y)<+\infty$.

Then any solution $\gamma$ of the Monge-Kantorovich's problem (1.2) with cost function (1.3) is induced by an optimal transportation map $T$, or equivalently $\gamma=(i d \times T)_{\sharp} \mu$, where $T$ is the solution of (1.1). 
Remark 3.2. The assumption ("for all $\gamma \in \Pi(\mu, \nu)$, and $\gamma$-a.e. $(x, y),\left(x, y^{\prime}\right), y \neq y^{\prime}$, then $x, y, y^{\prime}$ do not lie in a single line") on $\mu$ and $\nu$ shows that there are only two cases to transport mass located at point $x$. One is that the mass located at point $x$ is transferred to the only destination $y$, another is that the mass located at point $x$ is transferred to several possible destinations. In the second case, starting point $x$ and any two destinations $y, y^{\prime}$ do not lie in a single line. We shall give three examples to illustrate the idea. We recall a definition in advance.

Definition $3.3[7,10]$. The function $g(x)=\limsup _{r \rightarrow 0} \frac{\mu(B(x, r))}{\mathcal{L}^{n}(B(x, r))}$ is called density of a measure $\mu$, which is absolutely continuous with respect to $\mathcal{L}^{n}$. We denote it as $\mu=g \mathcal{L}^{n}$.

Example 3.4. Set $\mu=\frac{4}{\pi} \cdot 1_{B(0,1 / 2)} \mathcal{L}^{2}$ and $\nu(x)=\frac{1_{A}}{\mathcal{L}^{1}(A)} \mathcal{L}^{1}$, where $A=\left\{(1, y) \in \mathbb{R}^{2}:-1 \leq y \leq 1\right\}$. For every starting point $x \in B(0,1 / 2)$, either there is only one destination, or there are two destinations $u_{2}, u_{3} \in A$ and $x, u_{2}, u_{3}$ do not lie in a single line.

Example 3.5. Let $u_{i}, i=1,2,3$ be three points with coordinate $u_{1}=(1,0), u_{2}=(0,0), u_{3}=(-1,0)$. Set $\mu=\frac{4}{\pi} \cdot 1_{B\left(u_{2}, 1 / 2\right)} \mathcal{L}^{2}$ and $\nu=\frac{\delta_{u_{1}}+\delta_{u_{3}}}{2}$. For every starting point $x \in B\left(u_{2}, 1 / 2\right) \cap\left\{(x, y) \in \mathbb{R}^{2}: y=0\right\}$, either there is only one destination, or there are two destinations $u_{1}, u_{3}$ and $x, u_{1}, u_{3}$ do lie in a single line. In addition $\mathcal{L}^{2}\left(B\left(u_{2}, 1 / 2\right) \cap\left\{(x, y) \in \mathbb{R}^{2}: y=0\right\}\right)=0, \mu \ll \mathcal{L}^{2}$, then we get $\gamma-$ a.e. $(x, y),\left(x, y^{\prime}\right), y \neq y^{\prime}, x, y, y^{\prime}$ do not lie in a single line.

Example 3.6. Let $u_{i}, i=1,2$ be two points in $\mathbb{R}^{2}$, the distance between the two points satisfies $\left|u_{1}-u_{2}\right|>2$. Set $\mu, \nu$ probability measures absolutely continuous with respect to the Lebesgue measure with density $\frac{4}{\pi} \cdot 1_{B\left(u_{1}, 1 / 2\right)}$ and $\frac{4}{\pi} \cdot 1_{B\left(u_{2}, 1 / 2\right)}$. For every starting point $x \in B\left(u_{1}, 1 / 2\right)$, we can easily find two destinations $y, y^{\prime} \in B\left(u_{2}, 1 / 2\right)$, such that $x, y, y^{\prime}$ lie in a single line. (In fact, we can easily find many lines in which $x$ lies, and the lines intersect but not touch the ball $B\left(u_{2}, 1 / 2\right)$. Then the point $x$ and every two intersection points $y, y^{\prime} \in B\left(u_{2}, 1 / 2\right)$ do lie in a single line.)

In our paper, we deal with optimal transportation problems including Example 3.4 and 3.5, but not Example 3.6. In fact, if there are two destinations $y, y^{\prime}$ of $x$, and $y, y^{\prime}, x$ lie in a single line, then it is complicated to consider existence and uniqueness of optimal transportation maps (here the cost function is (1.3)). For example, we take the convex set $C$ big enough, then the transportation problem with cost function (1.3) is the same as the problem with a distance cost function. Then we have existence and non-uniqueness of optimal transportation maps in Example 3.6 (In fact, if both $\mu$ and $\nu$ are absolutely continuous with respect to the Lebesgue measure, and the cost function is the Euclidean distance, then there are solutions of the Monge's problem which are also solutions of the Kantorovich's problem, but no uniqueness is guaranteed, see [19].) Moreover, there may be no existence of optimal transportation maps, see examples in [1].

Remark 3.7. In Theorem 3.1, existence of solutions $\gamma$ to the Monge-Kantorovich's problem (1.2) comes from the weak compactness of $\Pi(\mu, \nu)$ and the lower semicontinuity of the cost function defined as (1.3). Denote by $R(\Gamma)$ the $\sigma$-compact set on which $\gamma$ is concentrated as in Lemma 2.7. In addition, the definition of the cost function defined as (1.3) and the optimality of $\gamma$ provide that $y-x \in C$ holds for $\gamma-$ a.e. $(x, y) \in R(\Gamma)$.

To prove Theorem 3.1, we need the following fundamental lemma.

Lemma 3.8. Let $\left(x_{0}, y_{0}\right)$ and $\left(x_{0}, y_{0}^{\prime}\right)$ two points of $R(\Gamma)$ and $x_{0} \neq y_{0}, x_{0} \neq y_{0}^{\prime}$. If

$$
\left\{(1-t)\left(y_{0}-x_{0}\right)+t\left(y_{0}^{\prime}-x_{0}\right): t \in[0,1]\right\} \cap \operatorname{Int}(C) \neq \emptyset,
$$

then $y_{0}=y_{0}^{\prime}$. 


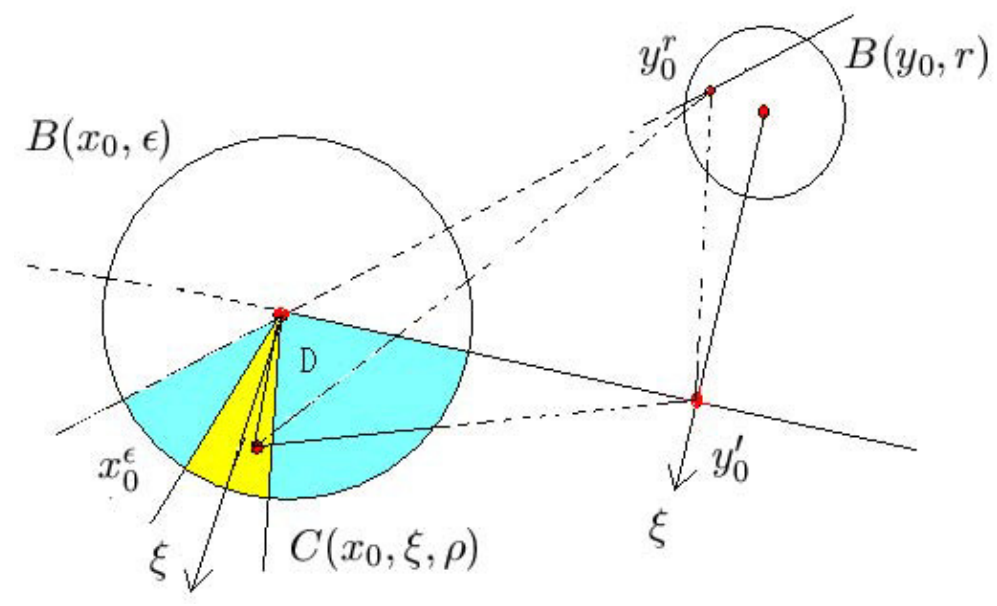

Figure 1. The position of $x_{0}^{\epsilon}, y_{0}^{r}, x_{0}, y_{0}^{\prime}$.

Before proving the lemma, we first state an assertion which is similar with the Monge's c-cyclical monotonicity in $\mathbb{R}^{2}[19,20]$. Let $\pi$ be an optimal transportation plan in the Monge problem with a Euclidean distance cost function in $\mathbb{R}^{2}$, and let $\left(x_{1}, y_{1}\right),\left(x_{2}, y_{2}\right) \in \operatorname{spt\pi }$ be every different two pairs. Denote by $[x, y]$ the line segment from $x$ to $y$. Then Monge's c-cyclical monotonicity is that either all four points $x_{1}, y_{1}, x_{2}, y_{2}$ lie in a single line or the two line segments $\left[x_{1}, y_{1}\right],\left[x_{2}, y_{2}\right]$ do not intersect, except that they may intersect at their endpoints, explicitly $\left[x_{1}, y_{1}\right]$ and $\left[x_{2}, y_{2}\right]$ may intersect at $x_{1}=x_{2}$ or $y_{1}=y_{2}$.

In our case, thanks to the definition of cost function (1.3), we get

Assertion: if $\left(x_{1}, y_{1}\right),\left(x_{2}, y_{2}\right) \in R(\Gamma)$ then the four points $x_{1}, y_{1}, x_{2}, y_{2}$ have the above c-cyclical monotonicity.

Proof of Lemma 3.8. To show this lemma, we proceed by contradiction. Let $\left(x_{0}, y_{0}\right)$ and $\left(x_{0}, y_{0}^{\prime}\right)$ be two points of $R(\Gamma)$ satisfying $y_{0} \neq y_{0}^{\prime}$. The assumptions on $\mu, \nu$ provide that $x_{0}, y_{0}, y_{0}^{\prime}$ do not lie in a single line.

Step 1. We can find a pair $\left(x_{0}^{\epsilon}, y_{0}^{r}\right)$ in $R(\Gamma)$, such that $x_{0}^{\epsilon}$ and $y_{0}^{r}$ are near $x_{0}$ and $y_{0}$ respectively. In addition, the pair $\left(x_{0}^{\epsilon}, y_{0}^{r}\right)$ satisfies the properties: $\frac{x_{0}^{\epsilon}-x_{0}}{\left|x_{0}^{\epsilon}-x_{0}\right|}$ and $\frac{y_{0}^{\prime}-y_{0}^{r}}{\left|y_{0}^{\prime}-y_{0}^{r}\right|}$ are close to $\frac{y_{0}^{\prime}-y_{0}}{\left|y_{0}^{\prime}-y_{0}\right|}$ (see Fig. 1), i.e. there exist $0<\rho<1$ and $0<r<1$ such that

$$
\left\langle\frac{x_{0}^{\epsilon}-x_{0}}{\left|x_{0}^{\epsilon}-x_{0}\right|}, \frac{y_{0}^{\prime}-y_{0}}{\left|y_{0}^{\prime}-y_{0}\right|}\right\rangle>1-\rho
$$

and

$$
\arcsin \frac{r}{\left|y_{0}^{\prime}-y_{0}\right|} \leq \arccos \left\langle\frac{y_{0}^{r}-y_{0}^{\prime}}{\left|y_{0}^{r}-y_{0}^{\prime}\right|}, \frac{y_{0}-y_{0}^{\prime}}{\left|y_{0}-y_{0}^{\prime}\right|}\right\rangle<\frac{\pi}{2}
$$

where $\langle$,$\rangle is the usual Euclidean inner product.$

We set $\xi=\frac{y_{0}^{\prime}-y_{0}}{\left|y_{0}^{\prime}-y_{0}\right|}$. The direction $\xi$ is an entering direction (see Lem. 2.9) at $y_{0}-x_{0}$ and $-\xi$ is an entering direction at $y_{0}^{\prime}-x_{0}$.

According to Lemma 2.9, let $\epsilon>0$ fixed which will be chosen later, then there exist $r>0$ and $\rho \in(0,1)$, such that:

$$
\begin{gathered}
C\left(y_{0}^{\prime}-x_{0},-\xi, \rho\right) \cap \overline{B\left(y_{0}^{\prime}-x_{0}, \epsilon\right)} \subset \operatorname{Int}(C) \\
\forall z \in B\left(y_{0}-x_{0}, r+\epsilon\right) \cap C, C(z, \xi, \rho) \cap \overline{B(z, \epsilon)} \subset \operatorname{Int}(C)
\end{gathered}
$$


Moreover, we can take $\rho$ and $r$ small enough, then we get

$$
\forall x \in C\left(x_{0}, \xi, \rho\right),\left\langle\frac{x-x_{0}}{\left|x-x_{0}\right|}, \frac{y_{0}^{\prime}-y_{0}}{\left|y_{0}^{\prime}-y_{0}\right|}\right\rangle>1-\rho
$$

and

$$
\forall y_{0}^{r} \in \overline{B\left(y_{0}, r\right)}, \arcsin \frac{r}{\left|y_{0}^{\prime}-y_{0}\right|} \leq \arccos \left\langle\frac{y_{0}^{\prime}-y_{0}^{r}}{\left|y_{0}^{\prime}-y_{0}^{r}\right|}, \frac{y_{0}^{\prime}-y_{0}}{\left|y_{0}^{\prime}-y_{0}\right|}\right\rangle<\frac{\pi}{2} .
$$

The two inequalities show that the directions of $x-x_{0}$ and $y_{0}^{\prime}-y_{0}^{r}$ are close to $\xi$.

In addition, Lemma 2.8 and $\left(x_{0}, y_{0}\right) \in R(\Gamma)$ show that we can choose a proper point $x_{0}^{\epsilon}$, such that

$$
x_{0}^{\epsilon} \in \gamma^{-1}\left(\overline{B\left(y_{0}, r\right)}\right) \cap \overline{B\left(x_{0}, \epsilon\right)} \cap C\left(x_{0}, \xi, \rho\right) .
$$

Then we can choose $y_{0}^{r} \in \overline{B\left(y_{0}, r\right)}$, so that the couple $\left(x_{0}^{\epsilon}, y_{0}^{r}\right) \in R(\Gamma)$ satisfy the distance and direction conditions at the beginning of this step.

Step 2. Furthermore, we can prove that the above pair $\left(x_{0}^{\epsilon}, y_{0}^{r}\right)$ in Step 1 satisfies $y_{0}^{\prime}-x_{0}^{\epsilon}, y_{0}^{r}-x_{0} \in \operatorname{Int}(C)$.

$\star$ To prove $y_{0}^{\prime}-x_{0}^{\epsilon}=y_{0}^{\prime}-x_{0}+\left(x_{0}-x_{0}^{\epsilon}\right) \in \operatorname{Int}(C)$.

In fact, $x_{0}^{\epsilon} \in B\left(x_{0}, \varepsilon\right)$ shows that $y_{0}^{\prime}-x_{0}^{\epsilon} \in B\left(y_{0}^{\prime}-x_{0}, \varepsilon\right)$. In addition, the direction of $x_{0}-x_{0}^{\epsilon}$ is close to $-\xi$ (see Step 1). As a result, $y_{0}^{\prime}-x_{0}^{\epsilon} \in C\left(y_{0}^{\prime}-x_{0},-\xi, \rho\right)$. Then $y_{0}^{\prime}-x_{0}^{\epsilon} \in B\left(y_{0}^{\prime}-x_{0}, \varepsilon\right) \cap C\left(y_{0}^{\prime}-x_{0},-\xi, \rho\right)$ and $y_{0}^{\prime}-x_{0}^{\epsilon} \in \operatorname{Int}(C)$ as a consequence of (3.1).

$\star$ To prove $y_{0}^{r}-x_{0} \in \operatorname{Int}(C)$.

Indeed, $x_{0}^{\epsilon}-x_{0} \in C(0, \xi, \rho) \cap B(0, \epsilon)$ shows that $y_{0}^{r}-x_{0}=\left(y_{0}^{r}-x_{0}^{\epsilon}\right)+\left(x_{0}^{\epsilon}-x_{0}\right) \in B\left(y_{0}^{r}-x_{0}^{\epsilon}, \varepsilon\right) \cap C\left(y_{0}^{r}-x_{0}^{\epsilon}, \xi, \rho\right)$. Furthermore, $y_{0}^{r}-x_{0}^{\epsilon}=\left(y_{0}-x_{0}\right)+\left(x_{0}-x_{0}^{\epsilon}\right)+\left(y_{0}^{r}-y_{0}\right)$ indicates that $y_{0}^{r}-x_{0}^{\epsilon} \in B\left(y_{0}-x_{0}, r+\epsilon\right)$. In addition, Remark 3.7 indicates that $y_{0}^{r}-x_{0}^{\epsilon} \in C$. Then (3.2) shows that $y_{0}^{r}-x_{0} \in \operatorname{Int}(C)$.

Step 3. Consider the positions of $x_{0}^{\epsilon}, y_{0}^{r}, x_{0}, y_{0}^{\prime}$.

We can choose $r$ small enough, such that

$$
\overline{B\left(y_{0}, r\right)} \cap\left[x_{0}, y_{0}^{\prime}\right]=\emptyset,
$$

then the three points $x_{0}, y_{0}^{r}, y_{0}^{\prime}$ do not lie in a single line, neither $x_{0}^{\epsilon}, y_{0}^{r}, x_{0}, y_{0}^{\prime}$ do (see Fig. 1).

The lines $x_{0} y_{0}^{\prime}$ and $x_{0} y_{0}^{r}$ divide $B\left(x_{0}, \epsilon\right)$ into four parts. Denote by $D \subset B\left(x_{0}, \epsilon\right)$ the part that every point $x \in D$ and $x_{0}, y_{0}^{r}, y_{0}^{\prime}$ construct a convex quadrangle (see Fig. 1).

Furthermore, we can take $\rho$ small enough such that $C\left(x_{0}, \xi, \rho\right) \cap B\left(x_{0}, \epsilon\right) \subset D$ (see Fig. 1). Since $x_{0}^{\epsilon} \in$ $C\left(x_{0}, \xi, \rho\right) \cap B\left(x_{0}, \epsilon\right) \subset D$ then $x_{0}^{\epsilon}, x_{0}, y_{0}^{r}, y_{0}^{\prime}$ construct a convex quadrangle. Observing $x_{0} \neq x_{0}^{\epsilon}$ and the directions of $x_{0}^{\epsilon}-x_{0}$ and $y_{0}^{r}-y_{0}^{\prime}$, we get that the line segments $\left[x_{0}^{\epsilon}, y_{0}^{r}\right]$ and $\left[x_{0}, y_{0}^{\prime}\right]$ intersect, but not at the endpoints $x_{0}^{\epsilon}=x_{0}$ and $y_{0}^{r}=y_{0}^{\prime}$. In fact $\left[x_{0}^{\epsilon}, y_{0}^{r}\right]$ and $\left[x_{0}, y_{0}^{\prime}\right]$ are diagonal lines of the convex quadrangle (see Fig. 1). Then we get a contradiction with the above assertion.

Now, we can proceed with the Proof of Theorem 3.1.

Proof of Theorem 3.1. As we mentioned in Remark 3.7, the direct method in the calculus of variations provides that there is an optimal transportation plan $\gamma$. Denote by $R(\Gamma)$ the $\sigma$-compact set on which $\gamma$ is concentrated as in Lemma 2.7. For two arbitrary pairs $\left(x_{0}, y_{0}\right),\left(x_{0}, y_{0}^{\prime}\right) \in R(\Gamma)$ satisfying $y_{0}-x_{0}, y_{0}^{\prime}-x_{0} \in C$, the strict convexity of $C$ provides that $\left\{(1-t)\left(y_{0}-x_{0}\right)+t\left(y_{0}^{\prime}-x_{0}\right): t \in[0,1]\right\} \cap \operatorname{Int}(C) \neq \emptyset$. As a result, Lemma 3.3 shows that $y_{0}=y_{0}^{\prime}$, i.e. $\gamma$ is induced by a transportation map. Theorem 2.3 shows that the transportation map is optimal. Then we get the desired result in Theorem 3.1.

Corollary 3.9. Under the assumption of Theorem 3.1, the solutions of (1.1) and (1.2) with cost function (1.3) are uniquely determined $\mu$-almost everywhere. 
Proof. The idea is classical: let $\gamma_{1}$ and $\gamma_{2}$ be two solutions of (1.2), and let $T_{1}$ and $T_{2}$ the associated transportation maps (given by Thm. 3.1). The probability measure $\frac{\gamma_{1}+\gamma_{2}}{2}$ is also an optimal transportation plan associated to an optimal transportation map $T_{3}$. Then (2.1) in Section 2 shows that for any $\varphi \in \mathcal{C}_{0}\left(\mathbb{R}^{2} \times \mathbb{R}^{2}\right)$,

$$
\begin{aligned}
\int_{\mathbb{R}^{2}} \varphi\left(x, T_{3}(x)\right) \mathrm{d} \mu(x) & =\int_{\mathbb{R}^{2} \times \mathbb{R}^{2}} \varphi(x, y) \mathrm{d} \frac{\gamma_{1}(x, y)+\gamma_{2}(x, y)}{2} \\
& =\frac{1}{2}\left(\int_{\mathbb{R}^{2} \times \mathbb{R}^{2}} \varphi(x, y) \mathrm{d} \gamma_{1}(x, y)+\int_{\mathbb{R}^{2} \times \mathbb{R}^{2}} \varphi(x, y) \mathrm{d} \gamma_{2}(x, y)\right) \\
& =\frac{1}{2}\left(\int_{\mathbb{R}^{2}} \varphi\left(x, T_{1}(x)\right) \mathrm{d} \mu(x)+\int_{\mathbb{R}^{2}} \varphi\left(x, T_{2}(x)\right) \mathrm{d} \mu(x)\right) .
\end{aligned}
$$

If we take $\varphi(x, y)=h(x) y$ where $h(x) \in \mathcal{C}_{0}\left(\mathbb{R}^{2}\right)$ is arbitrary, we have

$$
\int_{\mathbb{R}^{2}} h(x) \frac{T_{1}(x)+T_{2}(x)}{2} \mathrm{~d} \mu(x)=\int_{\mathbb{R}^{2}} h(x) T_{3}(x) \mathrm{d} \mu(x) .
$$

Since $h(x)$ is arbitrary, $T_{3}(x)=\frac{T_{1}(x)+T_{2}(x)}{2}$ holds $\mu-$ a.e. Taking the identity into the equation (3.3), we have

$$
\int_{\left\{x \in \mathbb{R}^{2}: T_{1}(x) \neq T_{2}(x)\right\}} \frac{\varphi\left(x, T_{1}(x)\right)+\varphi\left(x, T_{2}(x)\right)}{2} \mathrm{~d} \mu(x)=\int_{\left\{x \in \mathbb{R}^{2}: T_{1}(x) \neq T_{2}(x)\right\}} \varphi\left(x, T_{3}(x)\right) \mathrm{d} \mu(x) .
$$

Since $\varphi$ is arbitrary, by choosing $\varphi(x, y)=(x+y)^{2}$ we have $\mu\left(\left\{x \in \mathbb{R}^{2}: T_{1}(x) \neq T_{2}(x)\right\}\right)=0$, that is $\gamma_{1}=\gamma_{2}$ and $T_{1}=T_{2} \mu$ almost everywhere.

Generally speaking, for the original Monge's cost function, the minimizers of (1.1) are not unique. However we can give a sufficient condition for existence and uniqueness of solutions to the original Monge's problem with a Euclidean distance cost function in $\mathbb{R}^{2}$. In fact, existence and uniqueness results are the corollaries of our main Theorem 3.1 .

Theorem 3.10. Let $\mu \ll \mathcal{L}^{2}, \nu$ be two probability measures on $\mathbb{R}^{2}$, assume that

1. $\forall \gamma \in \Pi(\mu, \nu)$, and $\gamma-$ a.e. $(x, y),\left(x, y^{\prime}\right)$ satisfying $y \neq y^{\prime}$, then $x, y, y^{\prime}$ do not lie in a single line,

2. there exists $\pi \in \Pi(\mu, \nu)$ such that $\int_{\mathbb{R}^{2} \times \mathbb{R}^{2}}|x-y| \mathrm{d} \pi(x, y)<+\infty$,

then any solution $\gamma$ of the Monge-Kantorovich's problem

$$
\min _{\pi \in \Pi(\mu, \nu)} \int_{\mathbb{R}^{2} \times \mathbb{R}^{2}}|x-y| \mathrm{d} \pi(x, y)
$$

is induced by an optimal transportation map $T$, or equivalently $\gamma=(I d \times T)_{\sharp} \mu$.

Proof. The optimal transportation problem can be considered as an optimal transportation problem with a convex constraint $C=\mathbb{R}^{2}$, since $\mathbb{R}^{2}$ is strictly convex, then Theorem 3.1 provides existence of optimal transportation maps. In addition, uniqueness of optimal transportation maps comes from Corollary 3.9.

\section{Cost functions with COnvex COnstraints}

In this section, we consider convex constrained optimal transportation problems rather than strictly convex constrained ones. Using similar methods, we show there is an optimal transportation map of the MongeKantorovich problem where the cost function is the Euclidean distance with a convex constraint.

We begin with the following result which is a corollary of Lemma 3.6. 
Theorem 4.1. In Theorem 3.1, if $C$ is not strictly convex, then any solution of the Monge-Kantorovich problem with (1.3) is concentrated on a set $R(\Gamma)$ satisfying the following property: If $\left(x_{0}, y_{0}\right)$ and $\left(x_{0}, y_{0}^{\prime}\right)$ are different pairs in $R(\Gamma)$, then $\left(y_{0}-x_{0}\right)$ and $\left(y_{0}^{\prime}-x_{0}\right)$ belong to the same flat part of $C$.

Proof. We prove by contradiction. Assume that $\left(y_{0}-x_{0}\right)$ and $\left(y_{0}^{\prime}-x_{0}\right)$ do not belong to the same flat part of $C$, then we have

$$
\left\{(1-t)\left(y_{0}-x_{0}\right)+t\left(y_{0}^{\prime}-x_{0}\right): t \in[0,1]\right\} \cap \operatorname{Int}(C) \neq \emptyset .
$$

Lemma 3.6 shows that $y_{0}^{\prime}=y_{0}$. Then we get the contradiction and the desired result.

Theorem 4.2. Let $C$ be a closed convex set with at most countable flat parts, the other assumptions are the same as those in Theorem 3.1. Then there exists an optimal transportation map for the Monge-Kantorovich problem with the cost function defined as (1.3).

Proof. Denote by $C_{i}, i \in I$ the flat part of convex set $C$, and $C_{0}$ the set of points of $C$ which do not belong to any flat part of $C$. As a result

$$
C=\bigcup_{i \in I \cup\{0\}} C_{i}
$$

Existence of an optimal transportation plan comes from the weak compactness of the admissible set $\Pi(\mu, \nu)$ and the lower semicontinuity of (1.3) (the closeness of the convex set $C$ provides the lower semicontinuity). Denote by $\gamma$ an optimal transportation plan and $R(\Gamma)$ defined as Theorem 3.1 so that $\gamma$ is concentrated on $R(\Gamma)$. We will show that we can construct an optimal plan $\gamma$ which is concentrated on a graph.

Step 1. To prove that $\gamma$ can be decomposed as $\gamma=\left.\gamma\right|_{\Gamma_{0}}+\left.\sum_{i \in I} \gamma\right|_{\Gamma_{i}}$.

Let $\Gamma_{i}=\left\{(x, y): y-x \in C_{i} \cap R(\Gamma)\right\}, \quad i \in\{0\} \cup I$. For all $i \in I \cup\{0\}, \Gamma_{i}$ have the following property:

$$
(x, y) \in \Gamma_{i} \Rightarrow\left\{\left(x, y^{\prime}\right) \in R(\Gamma)\right\} \subset \Gamma_{i} .
$$

In fact, if $y=y^{\prime}$, then we get the result. If not, we prove by contradiction. We assume $\left(x, y^{\prime}\right) \notin \Gamma_{i}$ then we have

$$
\left\{t\left(y^{\prime}-x\right)+(1+t)(y-x): t \in[0,1]\right\} \cap \operatorname{Int}(C) \neq \emptyset .
$$

Lemma 3.6 tells us $y=y^{\prime}$, then we get a contradiction.

The above property shows that $\gamma=\left.\gamma\right|_{\Gamma_{0}}+\left.\sum_{i \in I} \gamma\right|_{\Gamma_{i}}$.

Then it is enough to construct an optimal plan which is induced by a map. In fact, by Theorem 3.1, the measure $\left.\gamma\right|_{\Gamma_{0}}$ is concentrated on a graph, and we need only to prove that $\forall i \in I,\left.\gamma\right|_{\Gamma_{i}}$ is induced by a map.

Step 2. To prove that for all $i \in I$, we can construct $\left.\gamma\right|_{\Gamma_{i}}$ which is induced by a map.

We denote $x=\left(x_{1}, x_{2}\right) \in \mathbb{R}^{2}$. For every $i \in I$ we can choose a coordinate system so that the equation of $C_{i}$ is $x_{2}=0$.

Then $y-x \in C_{i}$ implies $y_{2}-x_{2}=0$, so $\left.\gamma\right|_{\Gamma_{i}}$ is concentrated on

$$
\widetilde{\Gamma}:=\left\{(x, y) \in \mathbb{R}^{2} \times \mathbb{R}^{2}: x=\left(x_{1}, x_{2}\right), y=\left(y_{1}, y_{2}\right), x_{2}=y_{2}\right\} .
$$

We write

$$
\left.\gamma\right|_{\Gamma_{i}}\left(\left(x_{1}, x_{2}\right),\left(y_{1}, y_{2}\right)\right)=\widehat{\mu_{i}}\left(x_{1}, y_{1}\right) \times \zeta\left(x_{2}, y_{2}\right), \quad \text { with } \zeta:=\left.\pi_{x_{2}, y_{2} \sharp} \gamma\right|_{\Gamma_{i}},
$$

where $\pi_{x_{2}, y_{2}}(x, y)=\left(x_{2}, y_{2}\right)$. Then we have

$$
\begin{aligned}
\left.\int_{\mathbb{R}^{2} \times \mathbb{R}^{2}} c(x, y) \mathrm{d} \gamma\right|_{\Gamma_{i}} & =\left.\int_{\widetilde{\Gamma}}\left|x_{1}-y_{1}\right| \mathrm{d} \gamma\right|_{\Gamma_{i}} \\
& =\int_{\widetilde{\Gamma}}\left|x_{1}-y_{1}\right| \mathrm{d} \widehat{\mu}_{i}\left(x_{1}, y_{1}\right) d \zeta\left(x_{2}, y_{2}\right) .
\end{aligned}
$$


We set $\widehat{c}\left(x_{1}, y_{1}\right)=\left\{\begin{array}{cl}\left|x_{1}-y_{1}\right|, & \text { if }\left(y_{1}-x_{1}, 0\right) \in C_{i}, \\ +\infty, & \text { otherwise }\end{array}\right.$

On $\widetilde{\Gamma}$, for $\zeta$ - a.e. $\left(x_{2}, y_{2}\right)$, where $x_{2}=y_{2}$, we have:

1. $\widehat{f}_{0}^{i}=\pi_{x_{1} \sharp \widehat{\mu_{i}}}$ is absolutely continuous with respect to the Lebesgue measure $\mathcal{L}^{1}$, where $\pi_{x_{1}}\left(x_{1}, y_{1}\right)=x_{1}$, $\pi_{y_{1}}\left(x_{1}, y_{1}\right)=y_{1}$

2. let $\widehat{f}_{1}^{i}=\pi_{y_{1} \sharp \widehat{\mu_{i}}}$, the measure $\widehat{\mu}_{i}$ on $\mathbb{R}^{2}$ is a solution of the following transportation problem:

$$
\min \left\{\int_{\mathbb{R}^{2}} \widehat{c}\left(x_{1}, y_{1}\right) \mathrm{d} \pi\left(x_{1}, y_{1}\right): \pi \in \Pi\left(\widehat{f}_{0}, \widehat{f}_{1}\right)\right\} .
$$

The explicit proof of the above (1), (2) can be found in [12]. In addition, we need the following lemma to complete our proof.

Lemma 4.3 [12]. Let $\phi: \mathbb{R} \rightarrow \mathbb{R} \cup+\infty$ be a lower semicontinuous convex function (which means convex on the domain $\{x \in \mathbb{R}: \phi(x)<+\infty\}$ ), and $\mu, \nu$ be two probability measures on $\mathbb{R}$, with $\mu$ is absolutely continuous with Lebesgue measure $\mathcal{L}^{1}$. Then the optimal transportation problem (1.1) has a solution, but no uniqueness of optimal transportation maps is guaranteed.

Continued proof of Theorem 4.2. Lemma 4.3 provides that for all $i \in I$, the optimal transportation plan $\widehat{\mu_{i}}$ is induced by a map $\widehat{T}_{i}$, and then $\left.\gamma\right|_{\Gamma_{i}}$ is induced by a map $T_{i}$, where $T_{i}\left(x_{1}, x_{2}\right):=\left(\widehat{T}_{i} x_{1}, x_{2}\right)$. Furthermore we construct an optimal transportation plan $\gamma=\left.\gamma\right|_{\Gamma_{0}}+\left.\sum_{i \in I} \gamma\right|_{\Gamma_{i}}$ which is induced by a transportation map. Theorem 2.3 in Section 2 shows that the transportation map is optimal.

Remark 4.4. Lemma 4.3 shows that the uniqueness of $\widehat{T}_{i}$ can not be guaranteed. Furthermore, the optimal transportation maps $T_{i}$ are not unique. With the same method, we can construct an optimal plan $\gamma=\left.\gamma\right|_{\Gamma_{0}}+$ $\left.\sum_{i \in I} \gamma\right|_{\Gamma_{i}}$ which is induced by another transportation map, then there is no uniqueness of optimal transportation maps.

Acknowledgements. The first author would like to thank Professor Filippo Santambrogio for answering her questions via internet.

\section{REFERENCES}

[1] L. Ambrosio, Lectures notes on optimal transport problems. In Mathematical aspects of evolving interfaces, CIME summer school in Madeira (Pt), vol. 1812, edited by P. Colli and J. Rodrigues, Springer (2003) 1-52.

[2] L. Ambrosio and S. Rigot, Optimal mass transportation in the Heisenberg group. J. Funct. Anal. 208 (2004) $261-301$.

[3] S. Bianchini and F. Cavalletti, The monge problem in geodesic spaces. IMA Vol. Math. Appl. 153 (2011) $217-233$.

[4] L.A. Caffarelli, M. Feldman and R.J. McCann, Constructing optimal maps for Morge's transport problem as a limit of strictly convex costs. J. Amer. Math. Soc. 15 (2001) 1-26.

[5] G. Carlier, L. De Pascale and F. Santambrogio, A strategy for non-strictly convex transport cost and the example of $\|x-y\|^{p}$ in $\mathbb{R}^{2}$. Commun. Math. Sci. 8 (2010), 931-941.

[6] T. Champion and L. De Pascale, The monge problem for strictly convex norms in $\mathbb{R}^{d}$. J. Eur. Math. Soc. 12 (2010) $1355-1369$.

[7] T. Champion and L. De Pascale, The monge problem in $\mathbb{R}^{d}$. Duke Math. J. 157 (2011) 551-572.

[8] T. Champion, L. De Pascale and P. Juutinen, The $\infty-$ Wasserstein distance: local solutions and existence of optimal transport maps. SIAM J. Math. Anal. 40 (2008) 1-20.

[9] P. Chen, F. Jiang and X.-P. Yang, Optimal transportation in $\mathbb{R}^{n}$ for a distance cost with convex constraints. To appear.

[10] L. De Pascale and S. Rigot, Monge's transport problem in the Heisenberg group. Adv. Calc. Var. 4 (2010) 195-227.

[11] L.C. Evans and W. Gangbo, Differantial equations methods for the Monge-Kantorovich mass transfer problem. Mem. Amer. Math. Soc. 137 (1999) 1-66.

[12] C. Jimenez and F. Santambrogio, Optimal transportation for a quadratic cost with convex constrains and applications. J. Math. Pures Appl. 98 (2012) 103-113. 
[13] L.V. Kantorovich, On the translocation of masses. Dokl. Akad. Nauk. USSR 37 (1942) 199-201.

[14] L. Kantorovich, On a problem of Monge (in Russian). Uspekhi Mat. Nauk. 3 (1948) 225-226.

[15] R.J. McCann, N. Guillen, Five lectures on optimal transportation: geometry, regularity and applications. To appear in Proc. of the Séminaire de Mathématiques Supérieure (SMS) held in Montréal, QC, June 27-July 8, 2011.

[16] G. Monge, Mémoire sur la théorie des déblais et des Remblais. Histoire de l'Académie Royal des Sciences de Paris (1781) 666-704.

[17] V.N. Sudakov, Geometric problems in the theory of infinite-dimensional probability distributions. Proc. Steklov Inst. Math. $141(1979) 1-178$.

[18] N.S. Trudinger and X.-J. Wang, On the Monge mass transfer problem. Calc. Var. Partial Differ. Equ. 13 (2001) 19-31.

[19] C. Villani, Topics in optimal transportation. Graduate Studies in Mathematics, J. Amer. Math. Soc. Providence, RI 58 (2003).

[20] C. Villani, Optimal transport, old and new. Springer Verlag (2008). 